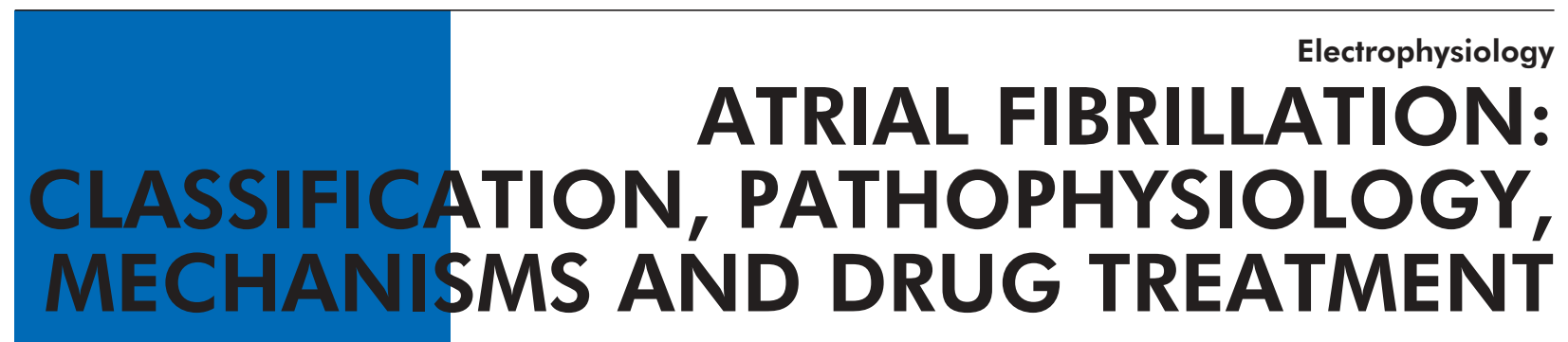

Vias Markides, Richard J Schilling

Heart 2003;89:939-943

T he prevalence of atrial fibrillation (AF), already the most common sustained cardiac arrhythmia, is constantly rising, even after adjusting for age and presence of structural heart disease. AF increases the risk of stroke sixfold and is associated with a twofold increase in mortality, which remains above 1.5-fold after adjusting for co-morbidity, predominantly caused by cerebrovascular events, progressive ventricular dysfunction, and increased coronary mortality. The adverse haemodynamic effects of AF are well described and relate not only to loss of atrial contraction, but also to the accompanying rapidity and irregularity of ventricular contraction. Although AF may be asymptomatic, up to two thirds of patients report that the arrhythmia is disruptive to their lives. Finally, the treatment of AF and its associated complications creates a significant and increasing economic burden. This article focuses predominantly on the pathophysiology of the arrhythmia and its pharmacological treatment. Anticoagulation for prevention of thromboembolism, a fundamental principle in the management of this arrhythmia, electrical cardioversion, percutaneous ablation techniques, and surgery for AF are not discussed in any detail.

\title{
CLASSIFICATION
}

AF may be classified based on aetiology, depending on whether it occurs without identifiable aetiology in patients with a structurally normal heart (lone AF), or whether it complicates hypertensive, valvar, or other structural heart disease.

A classification system based on the temporal pattern of the arrhythmia has been recently recommended. ${ }^{1}$ Patients presenting to medical attention may have a first detected episode of AF or, if previous episodes have been documented, recurrent arrhythmia. Episodes themselves may be paroxysmal, if they terminate spontaneously, usually within seven days, or persistent if the arrhythmia continues requiring electrical or pharmacological cardioversion for termination. AF that cannot be successfully terminated by cardioversion, and longstanding ( $>1$ year) AF, where cardioversion is not indicated or has not been attempted, is termed permanent (fig l).

\section{PATHOPHYSIOLOGY AND MECHANISMS}

Hypertensive, valvar, ischaemic, and other types of structural heart disease underlie most cases of persistent and permanent $\mathrm{AF}$, whereas lone $\mathrm{AF}$ accounts for approximately $15 \%$ of $\mathrm{AF}$ cases. Familial AF is well described, although at present considered rare. A region on chromosome 10 (10q22-q24) was originally identified as containing the gene responsible for AF in families in which the arrhythmia segregated as an autosomal dominant trait. However, familial AF appears to be a heterogeneous disease. A family with a mutation in the gene encoding the pore forming $\alpha$ subunit of the cardiac $I_{\mathrm{Ks}}$ channel on chromosome 11 that results in increased function of this channel, with affected members developing persistent AF probably caused by a reduction in refractoriness, has more recently been described. ${ }^{2}$

The pathogenesis of AF is now thought to involve an interaction between initiating triggers, often in the form of rapidly firing ectopic foci located inside one or more pulmonary veins, and an abnormal atrial tissue substrate capable of maintaining the arrhythmia. Although structural heart disease underlies many cases of AF, the pathogenesis of AF in apparently normal hearts is less well understood. Although there is considerable overlap, pulmonary vein triggers may play a dominant role in younger patients with relatively normal hearts and short paroxysms of AF, whereas an abnormal atrial tissue substrate may play a more important role in patients with structural heart

See end of article for authors' affiliations

Correspondence to: Dr Vias Markides, Waller Cardiac Department, St Mary's Hospital, Praed Street, London W2 INY, UK:

v.markides@imperial.ac.uk disease and persistent or permanent AF.

\section{Focal initiators of AF}

It is now known that foci of rapid ectopic activity, often located in muscular sleeves that extend from the left atrium into the proximal parts of pulmonary veins, play a pivotal role in the initiation of AF in humans. ${ }^{3}$ Less frequently, focal initiation of AF may be result from ectopic activity that arises from muscular sleeves in the proximal superior vena cava, from the ligament of Marshall, or other parts of the right and left atria. Initiation of AF by rapid focal activity has been demonstrated 


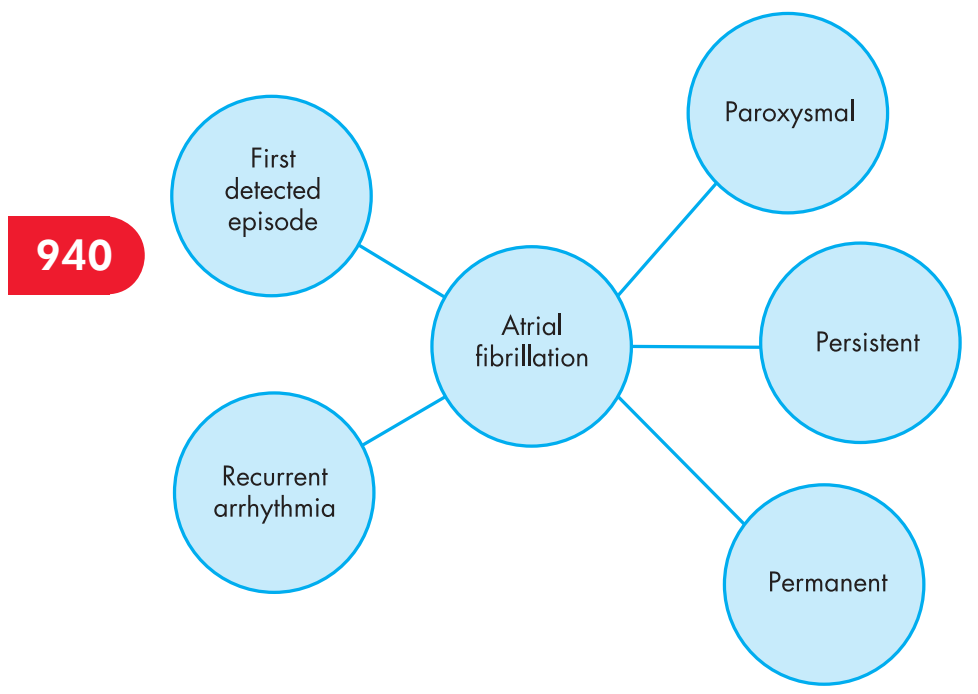

Figure 1 Temporal classification of atrial fibrillation (AF). An incident episode of AF presenting to medical attention may be the first ever detected episode of the arrhythmia, or represent recurrence of previously recognised arrhythmia (left). The episode may prove to be self terminating (paroxysmal), persistent (continuing until medical intervention such as DC cardioversion), or permanent (continuing for longer than one year or despite medical intervention such as DC cardioversion) (right).

not only in patients with structurally normal hearts and paroxysmal AF, but also during the process of reinitiation of persistent AF after electrical cardioversion, both in the presence and absence of associated structural heart disease. ${ }^{4}$

Muscular sleeves that extend into the proximal pulmonary veins are present in the normal heart. The mechanisms involved in the production of ectopic activity by these sleeves in patients with AF, as well as the exact mechanism of initiation of AF by the rapid activity, remain to be elucidated. Proposed mechanisms for generation of abnormal focus activity include increased automaticity, triggered activity, and micro-reentry. Changes in autonomic tone around the time of initiation of AF paroxysms, with an increase in sympathetic activity followed by an abrupt change to parasympathetic predominance, have also recently been demonstrated. ${ }^{5}$

\section{Tissue substrate capable of maintaining AF}

Both experimental and human mapping studies have demonstrated that persistent AF is generally characterised by the presence of multiple wavelets of excitation that propagate around the atrial myocardium. However, there is considerable variability in the observed patterns of activation, both between patients and between the two atria of individual patients. Perpetuation of $\mathrm{AF}$ is facilitated by the existence or development of an abnormal atrial tissue substrate capable of maintaining the arrhythmia, ${ }^{6}$ with the number of meandering wavelets that can be accommodated by the substrate determining the stability of $\mathrm{AF}^{78}$ Re-entry within the atrial myocardium is facilitated by conduction slowing and shortening of the refractory period. Both have been demonstrated in animal models and patients with $\mathrm{AF}$, with increased dispersion of refractoriness further contributing to arrhythmogenesis. Shortening of the atrial action potential, reduced expression of L type calcium channels, and microfibrosis of the atrial myocardium have also been demonstrated.

\section{Electrophysiological remodelling}

AF in itself can cause progressive changes in atrial electrophysiology such as substantial refractory period shortening,
Classification, pathophysiology, and mechanisms of AF: key points

- Atrial fibrillation (AF) is the most common sustained cardiac arrhythmia

- $\mathrm{AF}$ is usually classified according to its temporal pattern as paroxysmal, persistent, or permanent.

- AF adversely affects cardiac haemodynamics because of loss of atrial contraction and the rapidity and irregularity of the ventricular rate

- AF causes significant symptoms in approximately two thirds of patients

- $\mathrm{AF}$ is associated with a 1.5- to 2-fold increase in mortality

- $\mathrm{AF}$ is associated with a 6-fold increase in risk of stroke

- this risk can be substantially reduced with antithrombotic treatment

- decisions regarding antithrombotic treatment should not be based on the temporal pattern of the arrhythmia, but on the presence or absence of risk factors for thromboembolism in patients with AF

- $\mathrm{AF}$ is initiated by rapid electrical activity, often arising from arrhythmogenic foci located in the muscular sleeves of pulmonary veins. The arrhythmia is maintained by multiple re-entrant wavelets. Reduced refractoriness and conduction slowing facilitate re-entry

- After a period of continuous AF, electrical remodelling occurs, further facilitating AF maintenance (AF begets AF). These changes are initially reversible if sinus rhythm is restored, but may become permanent and be associated with structural changes if fibrillation is allowed to continue

which further facilitate perpetuation of the arrhythmia. In animal studies, changes in ion channel function and shortening of refractory periods start within minutes of AF onset and, by 24 hours, sufficient atrial remodelling has occurred to increase the likelihood of AF persisting. However, restoration of sinus rhythm in this animal model, even after two weeks of persistent AF, results in a rapid reversal of the electrophysiological remodelling. ${ }^{9}$

Electrical remodelling and its reversal also appear to occur in humans. Clinical observations, as well as a number of studies, have suggested that patients with recurrent AF may develop increasing problems with time and a significant proportion may progress to permanent AF. In patients undergoing electrical cardioversion of persistent AF, the duration of the antecedent episode is a potent predictor of maintenance of sinus rhythm. Moreover, patients with AF are at particularly high risk of recurrence of the arrhythmia in the first few days after cardioversion. ${ }^{10}$ Indeed, it has been demonstrated that shortened right atrial refractory periods observed immediately after cardioversion of persistent AF lengthen again within four weeks. ${ }^{11}$ Although reverse remodelling after restoration of sinus rhythm does occur in humans with established $\mathrm{AF}$, this may no longer be possible after very prolonged periods of $\mathrm{AF}^{12}$ and thus restoration and maintenance of sinus rhythm in these patients is often difficult.

\section{PHARMACOLOGICAL TREATMENT}

In patients with short paroxysms of AF, therapeutic strategies should generally concentrate on providing control of the arrhythmia itself. In patients with persistent $\mathrm{AF}$, however, the clinician is often faced with the dilemma as to whether to try and restore and then maintain sinus rhythm (rhythm control), or to accept the arrhythmia (as in the case of permanent AF) and control the ventricular rate (rate control). Regardless of the 


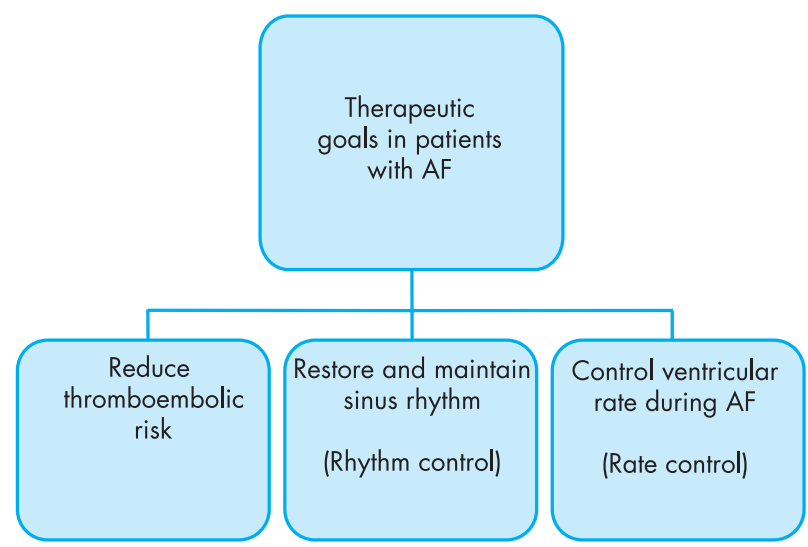

Figure 2 Therapeutic goals in patients with atrial fibrillation

arrhythmia pattern or the therapeutic strategy chosen, and in the absence of contraindications, patients should be considered for anticoagulation if they have one or more risk factors for thromboembolism (fig 2). Patients at low or intermediate risk, and higher risk patients in whom warfarin is contraindicated, may benefit from antiplatelet treatment. ${ }^{13}$

\section{Rate versus rhythm control}

There is still no consensus regarding whether patients with persistent AF are best managed using strategies that target the arrhythmia itself, or those that accept the arrhythmia and control the ventricular rate. With rate control strategies, the arrhythmia is allowed to continue, and symptomatic improvement is achieved solely because of better control of the ventricular rate. As the atria continue to fibrillate, the risk of thromboembolism persists and ventricular filling occurs only passively, without the active contribution of atrial contraction. Rhythm control, on the other hand, aims to restore sinus rhythm and thus synchronised atrioventricular contraction. In theory, this strategy should also help slow or prevent the progression to permanent AF and reduce the risk of thromboembolism, although there is as yet no evidence to support the latter assumption. Another important consideration, however, is the propensity for drugs used for rhythm control to cause serious proarrhythmia.

In a randomised open label pilot trial comparing rate control, predominantly using diltiazem, and rhythm control, predominantly using amiodarone with or without direct current (DC) cardioversion in patients with AF, the two strategies produced similar improvements in quality of life. A significant improvement in exercise tolerance as assessed by a six minute walk test was demonstrated in the rhythm control group, even though only $56 \%$ of the patients in this group achieved sinus rhythm. However, hospital admissions, predominantly for DC cardioversions, were higher in the rhythm control group. ${ }^{14}$

The results of the much larger AFFIRM (atrial fibrillation follow-up investigation of rhythm management) trial have recently been reported. ${ }^{15}$ The study enrolled more than 4000 patients with predominantly persistent AF. Enrolled patients (mean age 70 years) had at least one risk factor for stroke or death accompanying AF and could symptomatically tolerate the arrhythmia at baseline. Approximately $50 \%$ of patients randomised had a history of hypertension, whereas $25 \%$ had coronary artery disease or heart failure. Patients randomised to rate control received digoxin, $\beta$ blockers, or calcium antagonists, whereas those randomised to rhythm control received amiodarone, sotalol or propafenone and, if necessary, DC cardioversion. At follow up, sinus rhythm was achieved in only
$60 \%$ of patients in the rhythm arm, whereas satisfactory rate control was achieved in $80 \%$ of patients in the rate control arm. The primary end point of the study, all cause mortality, was not significantly different between the two groups, although there was a trend favouring rate control. There were also no differences in secondary end point components, including stroke rate, quality of life, or functional status and, although a trend favouring rate control was once again noted, anticoagulation was discontinued in more patients in the rhythm than in the rate control group. The majority of strokes in both groups occurred in patients with subtherapeutic levels of anticoagulation, or after warfarin had been stopped. In the pre-defined group of patients who were under the age of 65 , which accounted for approximately a quarter of patients included in the study, a trend favouring rhythm control was noted.

These results suggest that, at least in this elderly population of patients with AF and risk factors for stroke or death, rate control is at least as good as rhythm control. It should, however, be emphasised that these conclusions are not necessarily applicable to different patient populations, including younger patients with structurally normal hearts, or patients who are unable to tolerate the arrhythmia despite reasonable rate control. The results of AFFIRM also appear to be at odds with the results of a DIAMOND (Danish investigations of arrhythmia and mortality on dofetilide) substudy, in which patients (mean age 72 years) with heart failure or recent myocardial infarction and AF had been randomised to treatment with dofetilide or placebo. In this study, dofetilide was shown to be moderately effective at restoring sinus rhythm, but had no demonstrable effect on mortality. However, in a multivariate model, restoration of sinus rhythm, regardless of whether this was achieved pharmacologically, spontaneously, or electrically, was associated with a notable reduction in mortality. ${ }^{16}$

\section{Restoration of sinus rhythm}

Restoration of sinus rhythm in patients with AF may improve symptoms and cardiac haemodynamics, reverse the atrial remodelling associated with continuing arrhythmia, and, at least in theory, reduce the risk of thromboembolism. It has been demonstrated that restoration of sinus rhythm is associated with improvements in exercise capacity and peak oxygen consumption, both in patients with structural heart disease and in those with normal hearts. ${ }^{17}$

Since there is an important inverse association between duration of AF and likelihood of successful cardioversion or recurrence of arrhythmia, it is important that attempts to restore sinus rhythm are made as soon as this is possible and safe. However, although most guidelines suggest that cardioversion, be it pharmacological or electrical, within 48 hours of arrhythmia onset has a low risk of thromboembolism even without anticoagulation, the authors' policy is not to electively cardiovert patients who have been in AF without anticoagulation for longer than 12-24 hours.

For patients who have been in AF for longer, or in whom the duration of the arrhythmia is not clear, a minimum period of anticoagulation of three weeks is recommended before cardioversion. ${ }^{1}$ An alternative approach, particularly useful if there is clinical urgency to restore sinus rhythm, is to perform transoesophageal echocardiography in an attempt to exclude the presence of atrial thrombus before cardioversion. However, even if transoesophageal echocardiography has demonstrated 
no thrombus before cardioversion, patients must be anticoagulated for at least one month after cardioversion, since mechanical atrial function may return slowly after cardioversion.

Pharmacological cardioversion is often possible for the treatment of AF of recent onset, but efficacy is dramatically reduced in patients with AF that persists for more than 48 hours. Flecainide, administered intravenously in patients with AF of recent onset, has been shown to restore sinus rhythm in $72-95 \%$ of patients, with the greatest success rates in patients who receive treatment within 24 hours of AF onset. Flecainide also appears to be superior to both propafenone and amiodarone in this setting.

Pharmacological cardioversion is much less likely to be effective when AF has persisted for more than 48 hours. Administration of dofetilide to patients with persistent AF of more than two weeks' duration has been shown to restore sinus rhythm in $22-42 \%$ within three days. However, because of a significant risk of proarrhythmia, treatment must be initiated during continuous monitoring in hospital. Amiodarone appears to be the most effective agent for restoring sinus rhythm in patients with persistent AF, with one small study demonstrating sinus rhythm restoration in $44 \%$ and $68 \%$ of patients at two days and nine months, respectively. ${ }^{18}$ Electrical cardioversion, which has success rates between $65-90 \%$, is not discussed here.

\section{Sinus rhythm maintenance}

Flecainide and propafenone have been shown to be similarly effective at suppressing symptomatic paroxysms of AF and, in the absence of structural heart disease, neither drug appears to cause significant proarrhythmia. In general, these class Ic agents tend to be better tolerated and more effective than class Ia agents, such as quinidine and disopyramide.

Digoxin administration does not alter the probability of restoration or maintenance of sinus rhythm in patients with AF of recent onset. Pure $\beta$ adrenoceptor antagonists have a small beneficial effect in maintaining sinus rhythm in patients who have been cardioverted from AF. There appears to be no difference between pure $\beta 1$ antagonists and sotalol, either in reduction of AF burden in patients with paroxysmal AF, or in the likelihood of AF relapse after cardioversion, but an excess of proarrhythmic events has been noted in patients receiving sotalol. Sotalol may be better than propafenone at preventing AF paroxysms.

The efficacy of amiodarone has been demonstrated both in patients with paroxysmal AF and those with persistent AF refractory to other drugs, with a probability of arrhythmia suppression of $50-80 \%$ at $1-3$ years. In a direct comparison, amiodarone has more recently been shown to be superior to both propafenone and sotalol at maintaining sinus rhythm. ${ }^{19}$ An important consideration when prescribing amiodarone for long term treatment is that, in addition to its rare serious side effects, patients on amiodarone for long periods ( $>5$ years) frequently develop thyroid dysfunction.

Ultimately, the choice of pharmacological agent for sinus rhythm maintenance needs to be individualised, and based not only on the relative efficacy of the different agents, but also on their side effect profiles, contraindications, and the patient's ventricular function. $\beta$ Adrenoceptor antagonists may be preferred in patients with relatively normal hearts, with class Ic agents as an alternative, and amiodarone reserved for patients unresponsive to other drugs or those with poor ventricular function.

\section{Principles of AF management: key points}

- Assessment of thromboembolic risk and antithrombotic treatment for patients at risk

- A choice of:

- Restoration and maintenance of sinus rhythm (rhythm control)

- using electrical cardioversion, drugs, ablation, or surgery may be particularly useful in younger patients with structurally normal hearts and paroxysmal $\mathrm{AF}$, or persistent $\mathrm{AF}$ of recent onset

- surgery suitable even in long standing AF, but associated with substantial morbidity and mortality

- Acceptance of the arrhythmia and control of the ventricular rate (rate control)

- using drugs (usually $\beta$ or calcium channel blockers with or without digoxin), or occasionally atrioventricular node ablation and implantation of a permanent pacemaker

- may be more appropriate in elderly patients with hypertension or structural heart disease and persistent or permanent arrhythmia, especially if this can be tolerated symptomatically

\section{Ventricular rate control}

Digoxin is widely used for ventricular rate control during AF. Although generally safe to use even in patients with poor ventricular function, it appears to be less effective than other agents at controlling ventricular rate, particularly during acute or paroxysmal AF, exercise, or critical illnesses. The efficacy of digoxin at controlling the ventricular rate in $\mathrm{AF}$ is also limited during acute paroxysms of $\mathrm{AF}$, and use of the drug may prolong the duration of paroxysms. ${ }^{20}$ Diltiazem is effective at controlling ventricular rate in patients with $\mathrm{AF}$ and fast ventricular rates. Both diltiazem and verapamil are superior to digoxin at controlling ventricular rates during exercise and allow modest improvements in exercise capacity, without causing resting bradycardia or pauses. The benefits of calcium channel blockers as well as of $\beta$ blockers over digoxin appear to be particularly pronounced in patients with impaired diastolic filling, such as those with mitral stenosis. Combinations of digoxin with calcium channel blockers or $\beta$ blockers may not only improve ventricular rate control, both at rest and during exercise, but may also improve exercise capacity, even in patients with underlying ventricular dysfunction.

In patients with impaired ventricular function, chronic administration of amiodarone, in addition to reducing AF burden, significantly reduces the ventricular rate. Intravenous amiodarone may also be moderately effective at controlling the ventricular rate in critically ill patients with AF.

\section{Common mistakes}

Anticoagulation

In clinical practice, physicians are often less keen to prescribe anticoagulation for patients with paroxysmal AF than for those with persistent AF. Although the risk of thromboembolism may indeed be higher in patients with persistent $A F$, thromboembolic risk may be substantial even in patients with paroxysmal AF. Therefore decisions regarding anticoagulation should be predominantly based on the presence or absence of well established risk factors for thromboembolism, including previous stroke or transient ischaemic attack, valvar or other 
structural heart disease, hypertension, diabetes, age more than 65 years, and echocardiographic parameters such as left ventricular function and left atrial size, rather than on the temporal pattern of the disease.

\section{Rate control}

It is common for physicians to prescribe digoxin alone in attempts to control the ventricular response to AF. $\beta$ Blockers or calcium antagonists are more effective.

\section{Rhythm control}

It is also common for physicians to prescribe digoxin to cardiovert patients. Digoxin has no effect on the likelihood of cardioversion, whereas class I antiarrhythmic drugs or amiodarone are often effective.

\section{CONCLUSIONS}

$\mathrm{AF}$ is a common and increasingly prevalent arrhythmia that is associated with substantial morbidity and mortality. Because of the limited efficacy of catheter based treatments, especially for patients with persistent $\mathrm{AF}$, and the substantial morbidity and mortality associated with surgery for the arrhythmia, pharmacological therapy remains the mainstay of treatment for the majority of patients. The optimum treatment strategy for patients with persistent AF remains controversial, with some clinicians favouring rhythm control and others rate control. Ultimately, treatment needs to be individualised, based on symptomatology and the likelihood of maintenance of sinus rhythm. Regardless of these controversies in arrhythmia management, anticoagulation or antiplatelet therapy for stroke prevention form an integral part of treatment of patients with $\mathrm{AF}$ and risk factors for thromboembolism.

The predominant focus of recent developments in pharmacological therapy for AF has been the development of novel class III antiarrhythmic agents, each with characteristic effects on potassium channels. In general, these agents have proven moderately efficacious but carry a significant risk of proarrhythmia. While research in this field continues, other drugs such as specific serotonin receptor antagonists continue to be developed. Further developments in catheter ablation technologies may greatly facilitate safe isolation of multiple pulmonary veins for patients with predominantly paroxysmal $\mathrm{AF}$, whereas improvements in linear catheter ablation technologies, accompanied by three dimensional atrial mapping and catheter navigation, may facilitate creation of linear left atrial lesions, which appear to be critical for the successful treatment of patients with persistent arrhythmia.

\section{Authors' affiliations}

V Markides, St Mary's Hospital, London, UK

R J Schilling, St Bartholomew's Hospital, London, UK

\section{REFERENCES}

1 Fuster V, Ryden LE, Asinger RW, et al. ACC/AHA/ESC guidelines for the management of patients with atrial fibrillation: executive summary a report of the American College of Cardiology/American Heart Association task force on practice guidelines and the European Society of Cardiology committee for practice guidelines and policy conferences (committee to develop guidelines for the management of patients with atrial fibrillation) developed in collaboration with the North American Society of Pacing and Electrophysiology. Circulation 2001;104:2118-50.

- The definitive set of guidelines for the management of patients with AF.

2 Chen YH, Xu SJ, Bendahhou S, et al. KCNQ1 gain-of-function mutation in familial atrial fibrillation. Science $2003 ; 299: 251-4$
3 Haïssaguerre $M$, Jaïs $P$, Shah $D C$, et al. Spontaneous initiation of atrial fibrillation by ectopic beats originating in the pulmonary veins. N EnglJ Med 1998;339:659-66.

- The original publication that highlighted the significance of arrhythmogenic foci, often located in pulmonary veins, in the initiation of $A F$ and the efficacy of pulmonary vein ablation.

4 Lau CP, Tse HF, Ayers GM. Defibrillation-guided radiofrequency ablation of atrial fibrillation secondary to an atrial focus. J Am Coll Cardiol 1999:33:1217-26.

5 Bettoni M, Zimmermann M. Autonomic tone variations before the onset of paroxysmal atrial fibrillation. Circulation 2002;105:2753-9.

- A recent publication demonstrating the occurrence of significant changes in autonomic tone around the time of AF initiation.

6 Allessie MA, Lammers WJEP, Smeets JRLM, et al. Total mapping of atrial excitation during acetylcholine-induced atrial flutter and fibrillation in the isolated canine heart. In: Kulbertus HE, Olsson SB, Schlepper M, eds. Atrial fibrillation. Molndal, Sweden: Lindgren and Soner, 1982:44-62.

7 Allessie MA, Bonke FI, Schopman FJ. Circus movement in rabbit atrial muscle as a mechanism of tachycardia. III. The "leading circle" concept: a new model of circus movement in cardiac tissue without the involvement of an anatomical obstacle. Circ Res 1977:41:9-18.

8 Schilling RJ, Kadish AH, Peters NS, et al. Endocardial mapping of atrial fibrillation in the human right atrium using a non-contact catheter. Eur Heart J 2000;21:550-64.

9 Wijffels MC, Kirchhof CJ, Dorland R, et al. Atrial fibrillation begets atrial fibrillation. A study in awake chronically instrumented goats. Circulation 1995;92:1954-68.

- An important paper on the effects of atrial fibrillation and its reversal on the electrophysiological properties of the atria.

10 Tieleman RG, Van Gelder IC, Crijns HJ, et al. Early recurrences of atrial fibrillation after electrical cardioversion: a result of fibrillation-induced electrical remodeling of the atria? J Am Coll Cardiol 1998;31:167-73.

- This study demonstrates that there is a high incidence of AF recurrence in the first five days after cardioversion. It also suggests that drugs that lower the intracellular calcium concentration, such as $\beta$ blockers and calcium antagonists, may lower the rate of $A F$ recurrence if administered during $A F$ (before cardioversion).

11 Pandozi C, Bianconi L, Villani M, et al. Electrophysiological characteristics of the human atria after cardioversion of persistent atrial fibrillation. Circulation 1998;98:2860-5

12 Rodriguez LM, Timmermans C, Wellens HJ. Are electrophysiological changes induced by longer lasting atrial fibrillation reversible?: observations using the atrial defibrillator. Circulation 1999;100:113-6.

13 Albers GW, Dalen JE, Laupacis A, et al. Antithrombotic therapy in atrial fibrillation. Chest 2001;119:194S-206S.

- An in-depth review of antithrombotic treatment for patients with AF.

14 Hohnloser SH, Kuck KH, Lilienthal J. Rhythm or rate control in atrial fibrillation - pharmacological intervention in atrial fibrillation (PIAF): a randomised trial. Lancet 2000;356:1789-94.

15 Wyse DG, Waldo AL, DiMarco JP, et al. A comparison of rate control and rhythm control in patients with atrial fibrillation. N Engl J Med 2002; 347: 1825-33.

- The first large randomised trial comparing strategies to restore and maintain sinus rhythm versus accepting the arrhythmia and controlling the ventricular rate in patients with AF. There was no significant difference between the two groups, either in the primary end point of death or in a composite secondary end point that included death, disabling stroke, and major bleeding. Although a trend favouring rate control was noted, anticoagulation was more frequently stopped in the rhythm control group and the majority of strokes in both groups occurred in patients with subtherapeutic anticoagulation or after discontinuation of warfarin. A trend favouring rhythm control was observed in patients under the age of 65.

16 Pedersen OD, Bagger $\mathrm{H}$, Keller N, et al. Efficacy of dofetilide in the treatment of atrial fibrillation-flutter in patients with reduced left ventricular function: a Danish investigations of arrhythmia and mortality on dofetilide function: a Danish investigations of arrhythmia and
(Diamond) substudy. Circulation 2001;104:292-6.

- Among patients with cardiac failure enrolled in the DIAMOND study, a group of around $\mathbf{5 0 0}$ patients had atrial flutter or fibrillation at baseline. This substudy demonstrates the efficacy of dofetilide at restoring and maintaining sinus rhythm, as well as the risk of pro-arrhythmia. Although not a predefined end point, the study suggests that, at least in this group of patients, restoration of sinus rhythm, regardless of how it is achieved, is associated with reduced mortality.

17 Gosselink AT, Crijns HJ, van den Berg MP, et al. Functional capacity before and after cardioversion of atrial fibrillation: a controlled study. $\mathrm{Br}$ Heart J 1994;72:161-6.

18 Kerin NZ, Faitel K, Naini M. The efficacy of intravenous amiodarone for the conversion of chronic atrial fibrillation. Amiodarone vs quinidine for conversion of atrial fibrillation. Arch Intern Med 1996;156:49-53.

19 Roy D, Talajic M, Dorian P, et al. Amiodarone to prevent recurrence of atrial fibrillation. Canadian trial of atrial fibrillation investigators. N Engl J Med 2000;342:913-20.

20 Rawles JM, Metcalfe M, Jennings K. Time of occurrence, duration, and ventricular rate of paroxysmal atrial fibrillation: the effect of digoxin. $\mathrm{Br}$ Heart J 1990;63:225-7. 